\title{
Internal hernia of the small bowel through the sigmoid mesocolon
}

\begin{abstract}
A pre-operative diagnosis of an internal hernia of the small bowel through a defect of the sigmoid mesocolon is presented. The computed tomography (CT) demonstrated that the herniated small bowel traversed dorsal to the sigmoid colon to left lower abdomen, just like undermining through the sigmoid mesocolon. The CT findings and an illustrated figure corresponding to the $\mathrm{CT}$ pictures will be shown. A condition with similar appearance without hernia will also be described.
\end{abstract}

Keywords: internal hernia, transmesocolic hernia, ct
Volume 2 Issue 6 - 2017

\author{
Chung Kuao Chou \\ Department of Radiology, China
}

Correspondence: Chung Kuao Chou, Department of Radiology,Yuan's General Hospital, No. I62, Cheng-Kung I st. Rd, Kaohsiung, Taiwan, Republic of China, Tel 8867335 I I2I, Email wushungxian@kimo.com

Received: October 31, 2017 | Published: April 19, 2017
Abbreviations: PSB, proximal small bowel; HER, herniating point; PHSB, proximal herniated small bowel; DC, descending colon; PSC, proximal sigmoid colon; DSC, distal sigmoid colon; DHSB, distal herniated small bowel; P, psoas muscle; HSB, herniated small bowel; SB, small bowel

\section{Introduction}

Sigmoid-related hernia indicates small bowel protrusion either into the intersigmoid fossa (intersigmoid type), through a defect of the sigmoid mesocolon (transmesocolic type), or into the mesocolon itself (intramesocolic type) to left lower abdomen..$^{1-3}$ On CT, the herniated small bowel loops lie posterior and lateral to the sigmoid colon. The defect is located between the sigmoid colon and left psoas muscle or between the sigmoid loops. ${ }^{2,3}$ In the current report, beside the characteristic CT manifestations of transmesocolic hernia, another condition with similar relationship of the small bowel and sigmoid colon on axial images without hernia will also be discussed.

\section{Case presentation}

A 55year-old male consulted to our emergency department with the complaint of a sudden-onset upper abdominal pain at about $6 \mathrm{pm}$ today. The routine blood and biochemical data were non-specific. He was just discharged from the surgical ward of our hospital after a laparoscopic partial descending colectomy for colonic carcinoma 11 days ago. About $12 \mathrm{~cm}$ long descending colon was resected. The pTNM stage was T1N0M0 and clinical stage I. An emergent CT examination demonstrated a long small bowel segment traversing dorsal to the sigmoid colon to left lower abdomen (Figures 1A-1C \& $2 \mathrm{~A}-2 \mathrm{C}$ ). The proximal small bowel was dilated and abruptly narrowed, with a beak-like or triangular-shape appearance, at a site dorsal to the rational course of the sigmoid colon. It seemed that this segment was undermining through a hole in the sigmoid mesocolon. The associated mesenteric vessels also showed a converging appearance like they were passing through a tunnel on the coronal images (Figure 2A-2C). Under the impression of internal hernia of the small bowel through the sigmoid mesocolon (transmesocolic hernia), the patient was admitted to receive further treatment. Because of progression of abdominal distension, a second laparoscopic operation was performed 5days later. During operation, a long small bowel segment herniated through a defect, which was about $10 \mathrm{~cm}$ long, of the sigmoid mesocolon was noted. The small bowel proximal to the herniation was dilated, consistent with clinical presentation of obstruction. The herniated small bowel was pulled back without irreversible damage and the defect repaired. The patient recovered uneventfully and discharged 1week later after operation.

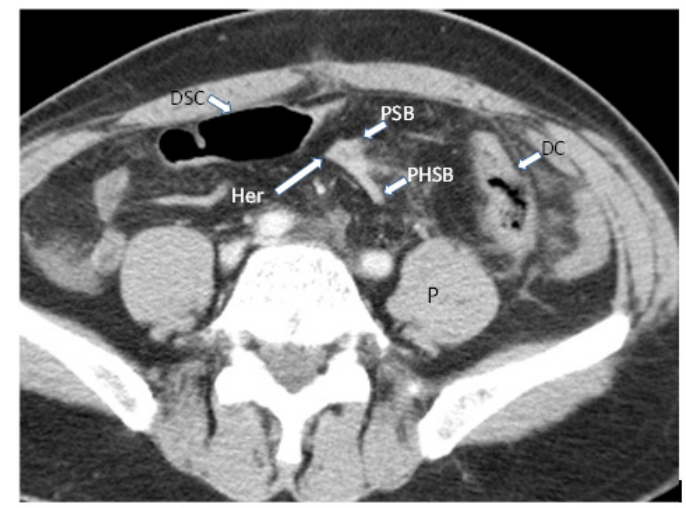

Figure IA Post-contrast axial image. The proximal small bowel (PSB) at the herniating point (her) assumed a beak- or triangular-shape and continued to proximal herniated small bowel (PHSB). It was located dorsal to proximal sigmoid colon (shown on IB) which was connected to descending (DC) and distal sigmoid colon (DSC). P-psoas muscle.



Figure IB Axial image at a level $2 \mathrm{~cm}$ caudal to IA. The proximal sigmoid colon (PSC) lay in front of the proximal (PHSB) and distal (DHSB) herniated small bowel. DSC-distal sigmoid colon. P-psoas muscle. 




Figure ICA diagram shows two levels, straight lines IA and IB, corresponding to images levels shown in Figures IA \& IB. HSB-herniated small bowel.



Figure 2A Reformatted coronal image. The most anterior image showed the anteriorly displaced proximal sigmoid colon (PSC).

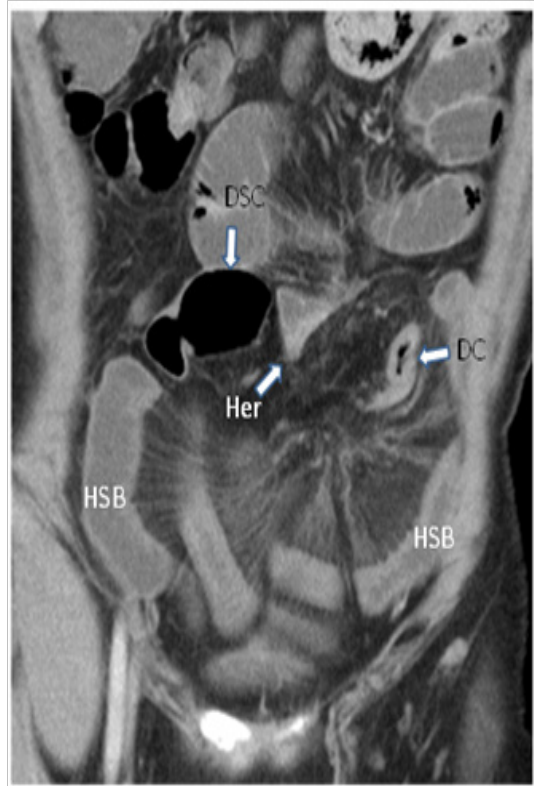

Figure 2B An image $15 \mathrm{~mm}$ posterior to Figure $2 \mathrm{~A}$ showed the beak-like or triangular-shaped herniating point (Her), descending colon (DC), distal sigmoid colon (DSC), and fan-distributed herniated small bowel (HSB). The herniating point was posterior to the proximal sigmoid colon shown on Figure 2A. The herniated small bowel mesentery showed edematous appearance.

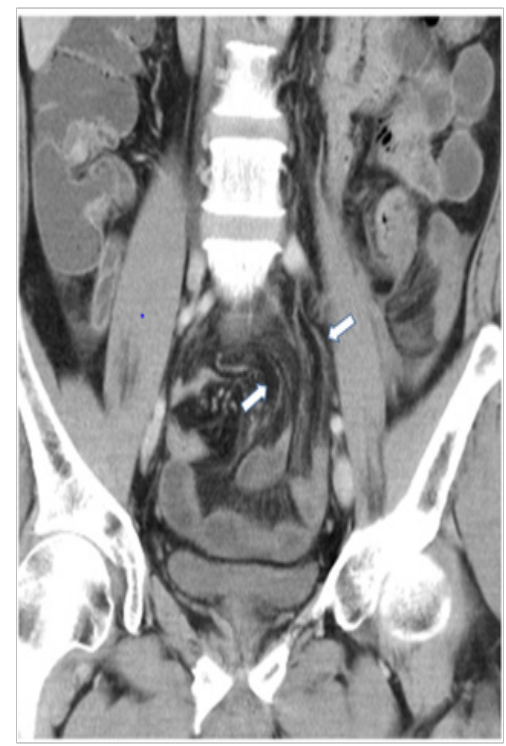

Figure $2 \mathrm{C}$ An image $40 \mathrm{~mm}$ posterior to Figure $2 \mathrm{~B}$ showed vascular converging appearance (arrows) like traversing through a tunnel.

\section{Discussion}

Internal hernia is protrusion of the viscera through normal or abnormal apertures into another compartment of the abdominal cavity. These apertures include normal foramina, recesses or fossae; congenital, surgery- or trauma-induced defect; or adhesion-induced foramen. ${ }^{1-3}$ Its clinical presentations include nausea, vomiting, mild to severe abdominal pain, and abdominal distension. The outcomes range from spontaneous recovery, obstruction, to catastrophic bowel necrosis. The common CT appearances of internal hernias include two closely opposed ends of a dilated small bowel segment, radially distributed U- or C-shaped bowel loops, vascular convergence, special relationship of the splanchnic vessels and adjacent bowels, and a circumscribed mass or special location of the herniated small bowel..$^{2-11}$

Among these internal hernias, the sigmoid-related hernia occupies about $6 \% .{ }^{3}$ The characteristic $\mathrm{CT}$ findings of this hernia is protrusion of the small bowel loops between the sigmoid colon and left psoas muscle to lie posterior and lateral to the sigmoid colon on the transverse plane. ${ }^{2,3}$ However, the small bowel may lie in a similar location on axial images of CT without transmesocolic hernia (Figure 3A-3C). In most conditions, the retroperitoneal descending colon is anchored parallel to the vertebral column on lateral side of retroperitoneum by the dorsal parietal peritoneum, and the intraperitoneal sigmoid mesocolon anchored obliquely on infero-lateral side of the retroperitoneum. Thus the small bowel always lies anterior and medial to the descendingsigmoid colon. However, the descending colon is not uncommonly found to move close to the midline and the sigmoid colon is dragged to right lower abdomen. In that circumstance, the small bowel may overlie in front of the descending-sigmoid colon to left lower abdomen and locate posterior and lateral to the sigmoid colon on the axial images, mimicking that of transmesocolic hernia. The critical difference between these two conditions is that the small bowel traverses dorsal to the sigmoid colon in transmesocoilic hernia. In the present case, by following the images sequentially, the relationship between the herniated small bowel loops and descending-sigmoid colon was correctly ascertained as undermining of the small bowel through the sigmoid mesocolon. The mesocolic defect was supposed to be secondary to the laparoscopic operation 11days ago. 




Figure 3A A diaphragm shows a condition which may mimic transmesocolic hernia of the small bowel on axial images. The descending colon lies close to midline and the sigmoid colon shifts to right lower abdomen. The small bowe (SB) then overlies the descending-sigmoid colon to a location posterior and lateral to the sigmoid colon on axial images. The straight lines $3 B$ and $3 C$ represent two levels corresponding to Figure 3B \& 3C.



Figure 3B The descending colon (DC) is close to the midline. The normal small bowel (SB) and mesenteric vessels (arrow) overlie in front of the descending colon to left side.

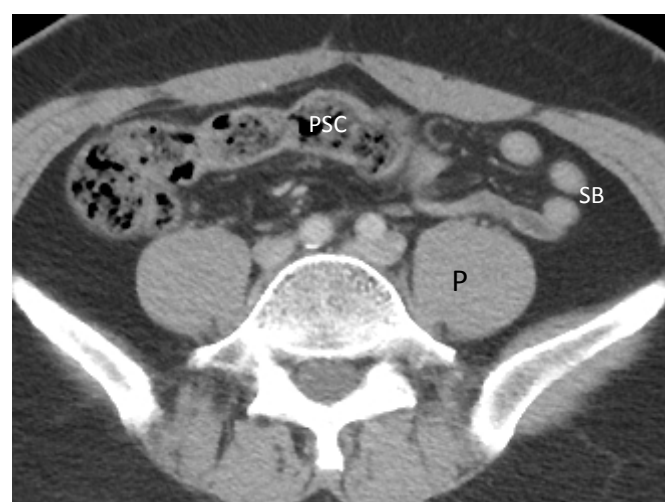

Figure 3C The normal small bowel (SB) lies posterior and lateral to the proximal sigmoid colon (PSC), and between the PSC and psoas muscle (P).

\section{Conclusion}

Identification of a transmesocolic hernia of the small bowel on CT needs recognition of not only a characteristic location of the small bowel which is posterior and lateral to the sigmoid colon, but also the abnormal course of the herniated small bowel and the associated vessels which should be dorsal to the sigmoid colon. A similar location of the small bowel on axial images without hernia may be seen in the case of medially displaced descending colon. In that condition, the small bowel and its associated vessels overlie in front of, instead of dorsal to, the descending-sigmoid colon.

\section{Acknowledgements}

None.

\section{Conflict of interest}

Author declares that there is no conflict of interest.

\section{References}

1. Mayers MA. Internal abdominal hernias. In: Mayers MA, editor. Dynamic radiology of the abdomen. 5th ed. New York: Springer-Verlag; 2000. p. 711-748.

2. Yu CY, Lin CC, Yu JC, et al. Strangulated transmesosigmoid hernia: CT diagnosis. Abdom Imaging. 2004;29(2):158-160.

3. Martin LC, Merkle EM, Thompson WM. Review of Internal Hernias: Radiographic and Clinical Findings. AJR Am J Roentgenol. 2006;186(3):703-717.

4. Balthazar EJ. CT of small bowel obstruction. AJR Am J Roentgenol. 1994;162:255-261.

5. BalthazarEJ, Liebeskind ME, Macari M. Intestinal ischemia in patients in whom small bowel obstruction is suspected: evaluation of accuracy, limitations, and clinical implications of CT in diagnosis. Radiology. 1997;205(2):519-522.

6. OlazabalA, Guasch I, Casas D. CT diagnosis of nonobstructive left paraduodenal hernia. Clin Radiol. 1992;46(4):288-289.

7. InoueY, Nakamura H, Mizumoto S, et al. Lesser sac hernia through the gastrocolic ligament: CT diagnosis. Abdom Imaging. 1996;21(2):145-147.

8. DelabrousseE, Couvreur M, Saguet O, et al. Strangulated transomental hernia: CT findings. Abdom Imaging. 2001;26(1):86-88.

9. Chou CK, Mal CW, Wu RH, et al. Combined Transmesocolic-Transomental Internal Hernia. AJR Am J Roentgenol. 2005;184(5):1532-1534.

10. Takeyama N, Gokan T, Ohgiya Y, et al. CT of internal hernia. RadioGraphics. 2005;25(4):997-1015.

11. Marrouai W, Petitcolin V, Bros S, et al. Internal hernia of the broad ligament: CT diagnosis for laparoscopic management. Diagn Interv Imaging. 2012;93(7-8):621-624. 